\title{
Near-Optimum Soft-Output Ant-Colony-Optimization Based Multiuser Detection for the DS-CDMA Uplink
}

\author{
Chong Xu, Lie-Liang Yang, Rob Maunder and Lajos Hanzo \\ School of ECS., Univ. of Southampton, SO17 1BJ, UK. \\ Tel: +44-23-80-593 125, Fax: +44-23-80-593 045 \\ Email: \{cx05r, 1ly, lh\}@ecs.soton.ac.uk, http://www-mobile.ecs.soton.ac.uk
}

\begin{abstract}
In this contribution, a novel soft-output Ant Colony Optimization (ACO) based Multi-User Detector (MUD) is proposed for the synchronous Direct-Sequence Code-DivisionMultiple-Access (DS-CDMA) uplink. The foraging behaviour of the ant colony in nature motivates the employment of reducedsearch ACO-based MUDs, which are capable of approaching the optimum Maximum Likelihood (ML) MUD's performance at the cost of a computational complexity, which maybe as low as that of the Mathched Filter (MF) based Single User Detector (SUD). However, the previously proposed conventional ACO based MUDs were unable to provide soft Log-Likelihood Ratio (LLR) values for the channel decoder. Hence in this paper, we present a novel soft-output ACO-MUD capable of delivering soft LLRs, which allows a CDMA system to achieve a near-single-user performance without any additional information feedback from the channel decoder, even when the number of users supported is as high as the number of chip in the spreading sequence. Our numerical results show that at a BER of $10^{-3}$, the performance of the currently known ACO-assisted state-of-the-art systems can be improved by about $17 \mathrm{~dB}$ with the aid of the proposed soft-output ACO-MUD. More explicitly, the soft-output ACOMUD is capable of approaching the optimum performance of the Bayesian detector, when $K=32$ UL users are supported with the aid of 31-chip Gold codes, while the complexity of the former is only a fraction of $10^{-8}$ of the latter.
\end{abstract}

\section{INTRODUCTION}

Ant Colony Optimization (ACO) was inspired by studying the foraging behavior of ants. It was first invoked by Colorni, Dorigo and Maniezzo [1], [2] in 1991. This population-based approach has recently been applied to a large number of so-called Nondeterministic Polynomial (NP)-hard combinatorial optimization problems, such as the Traveling Salesman Problem (TSP) [1], [3]-[5] and the Quadratic Assignment Problem (QAP) [3], [4], [6], the Job-Shop Scheduling Problem (JSP) [4], [7] as well as in the packet routing algorithms of telecommunication networks [8]-[10]. These investigations motivated its employment in near-Maximum Likelihood (ML) Multi-User Detection (MUD) aided Multiple Access (MA) systems [11]-[17].

The ACO framework defined by Marco [18] comprises a number of algorithms, including the 'ant system' [1], [4], the

Acknowledgements: The financial support of the EPSRC, UK of the Vodafone DHPA, and that of the EU is gratefully acknowledged. 'ant colony system' [5] and the 'MAX-MIN ant system' [3], [19]. The authors of [15]-[17] employed the 'ant colony system' [5], [20] to simplify the exhaustive search imposed by the optimum ML detector, while approaching the Bit-ErrorRatio (BER) performance of the latter. On the other hand, the authors of [11]-[14] developed a slightly different near-ML MUD. The ACO based MUD of both [15]-[17] and of [11][14] are capable of achieving a lower BER as well as a lower complexity than the Genetic Algorithm (GA)-based MUDs of [21], [22]. The authors of [16], [17] achieved a near-ML BER performance in the context of various Multi-Carrier (MC) DS-CDMA systems employing 31-chip Gold codes as the Tdomain spreading sequence, while supporting $K=32$ users. The required number of FLoating point Operations Per Second (FLOPS) was a factor of $10^{8}$ lower than that of the ML MUD.

However, to the best of the authors' knowledge, the ACObased MUD schemes found in the open literature at the time of writing are only capable of providing hard-decision output for the channel decoder. Against this background, in this contribution we present a novel soft-output ACO-MUD and quantify both its performance as well as its complexity in a Turbo Convolutional (TC) coded CDMA UL system. We will demonstrate that the DS-CDMA UL system exploiting the ACO's soft outputs is capable of outperforming its counterpart based on hard-decision outputs, when $K=32$ users are supported using 31chip-Gold codes. Our simulation results will demonstrate that the soft-output ACO assisted DS-CDMA UL achieves a similar BER performance to that attained by the Bayesian detector, while the complexity of the former one is only a fraction of the latter.

The rest of this paper is organized as follows. The model of the soft-output ACO assisted DS-CDMA UL system will be characterized in Section II. The conventional ACO-MUD is briefly reviewed in Section III. In Section IV, our new softoutput ACO-MUD is detailed. Our simulation results will be provided in Section V. Finally, we will conclude our discourse in Section VI.

\section{SYSTEM DESCRIPTION}

Fig. 1 shows the UpLink (UL) DS-CDMA system model, where each of the $K$ UL transmitters and the Base Station 


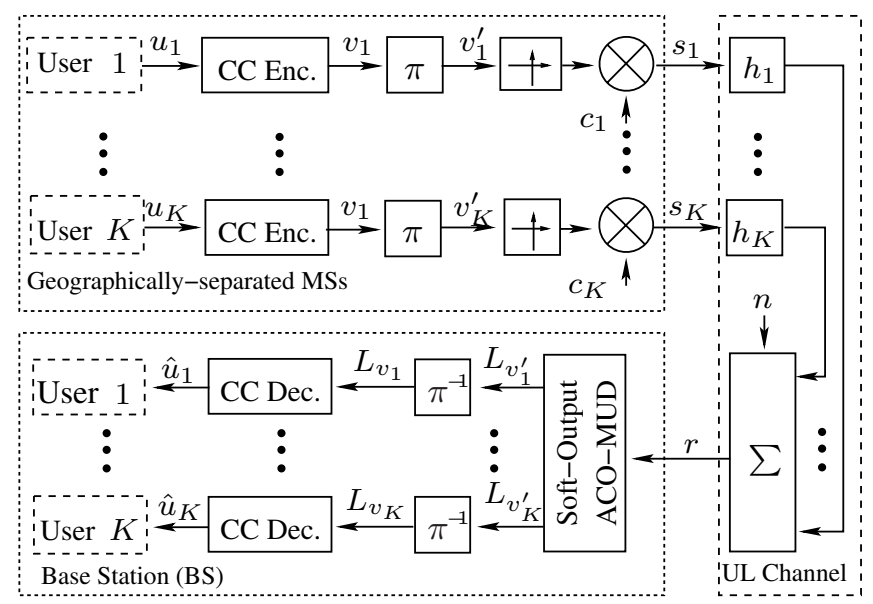

Fig. 1: Schematic of the soft-output ACO assisted DS-CDMA UL transceiver, where $K$ users are supported.

(BS) receiver employ a single transmit and receive antenna. The $k$ th user's $N_{u}$-bit data sequence $\left\{u_{k}[i] ; i=1, \ldots, N_{u}\right\}$ is firstly channel encoded at a rate of $R_{c}=N_{u} / N_{c}$, yielding the coded sequence $\left\{v_{k}[i] ; i=1, \ldots, N_{c}\right\}$ having a length of $N_{c}$ bits. This sequence is then interleaved by the random bitinterleaver $\pi$ of Fig. 1, generating the interleaved bit sequence $\left\{v_{k}^{\prime}[i] ; i=1, \ldots, N_{c}\right\}$, which is then fed to a buffer having a memory of $N_{c}$ bits. As seen from Fig. 1, the signal processing in the inner encoder and decoder is carried out on a bit-bybit basis. Therefore, we omit the bit index $i$ for the sake of brevity in these two components. The output of the buffer is then spread employing a user-specific $N_{s}$-chip DS spreading sequence waveform $c_{k}(t)$. Then, the DS spread signal will be Binary-Phase-Shift-Keying (BPSK) modulated at a carrier frequency $f$ that is common for all the users. Thus the transmitted signal of user $k$ can be expressed as:

$$
s_{k}(t)=\sqrt{2} v_{k}^{\prime} c_{k}(t) \cos \left(2 \pi f t+\phi_{k}\right),
$$

where each user is assumed to have a unity transmit power and $\phi_{k}$ is the phase angle introduced in the carrier modulation process. The modulated signal $s_{k}$ then propagates from the $k$ th MS's UL transmit antenna to the BS's receive antenna over a single-path flat Rayleigh fading channel $h_{k}$, which is assumed to be constant over a symbol duration. During the $n_{s}$ th chip duration, where we have $n_{s}=1, \ldots, N_{s}$, the received signal $r_{n_{s}}$ is the superposition of the signals transmitted from the $K$ MSs, which is also contaminated by the Additive White Gaussian Noise (AWGN). Thus, during a symbol interval, the $\left(N_{s} \times 1\right)$-element base-band equivalent received signal vector corresponding to the signals received during the $N_{s}$ chip durations can be expressed as:

$$
\mathbf{r}=\mathbf{C H v}^{\prime}+\mathbf{n},
$$

where we introduced the $\left(N_{s} \times 1\right)$-element base-band equivalent received signal vector $\mathbf{r}$ associated with the $N_{s}$ chip durations, the $(K \times K)$-element complex-valued CHannel Transfer Function $(\mathrm{CHTF})$ matrix $\mathbf{H}$, the $(K \times 1)$-element base-band equivalent transmitted signal vector $\mathbf{v}^{\prime}$ related to the $K$ users, the $\left(N_{s} \times 1\right)$-element AWGN noise vector $\mathbf{n}$ and the $\left(N_{s} \times K\right)$-element Gold-code matrix $\mathbf{C}$ as follows:

$$
\begin{aligned}
& \mathbf{r}=\left[\begin{array}{llll}
r_{1} & r_{2} & \ldots & r_{N_{s}}
\end{array}\right]^{T}, \\
& \mathbf{H}=\operatorname{diag}\left\{\left[\begin{array}{llll}
h_{1} & h_{2} & \cdots & h_{K}
\end{array}\right]\right\} \text {, } \\
& \mathbf{v}^{\prime}=\left[\begin{array}{llll}
v_{1}^{\prime} & v_{2}^{\prime} & \ldots & v_{K}^{\prime}
\end{array}\right]^{T}, \\
& \mathbf{n}=\left[\begin{array}{llll}
n_{1} & n_{2} & \ldots & n_{N_{s}}
\end{array}\right]^{T}, \\
& \mathbf{C}=\left[\begin{array}{cccc}
c_{11} & c_{21} & \cdots & c_{K 1} \\
c_{12} & c_{22} & \cdots & c_{K 2} \\
\cdots & \ldots & \ddots & \ldots \\
c_{1 N_{s}} & c_{2 N_{s}} & \cdots & c_{K N_{s}}
\end{array}\right] .
\end{aligned}
$$

Note that each element in $\mathbf{n}$ has a mean of zero and a variance of $\sigma_{n}^{2}$. For simplicity, we assume that the CHTFs are perfectly known at the BS. It can be shown that the Maximum-RatioCombining (MRC) based Matched Filter's (MF) output vector corresponding to the $K$ users can be expressed as:

$$
\begin{aligned}
\mathbf{y} & =(\mathbf{C H})^{H} \mathbf{r}=\mathbf{H}^{H} \mathbf{C}^{T} \mathbf{C H \mathbf { v } ^ { \prime }}+\mathbf{H}^{H} \mathbf{C}^{T} \mathbf{n} \\
& =\mathbf{R} \mathbf{v}^{\prime}+\tilde{\mathbf{n}},
\end{aligned}
$$

where we have:

$$
\begin{aligned}
\mathbf{y} & =\left[\begin{array}{llll}
y_{1} & y_{2} & \cdots & y_{K}
\end{array}\right]^{T}, \\
\mathbf{R} & =\mathbf{H}^{H} \mathbf{C}^{T} \mathbf{C H} .
\end{aligned}
$$

\begin{tabular}{|c|c|c|c|c|}
\hline & 1 & 2 & . & $K$ \\
\hline & $v_{1}^{\prime}=+1$ & $v_{2}^{\prime}=+1$ & . & $v_{K}^{\prime}=+1$ \\
\hline & $v_{1}^{\prime}=-1$ & $v_{2}^{\prime}=-1$ & . & $v_{K}^{\prime}=-1$ \\
\hline
\end{tabular}

\section{Conventional Ant Colony Optimization Based MultiUser Detector}

In both the conventional or the proposed soft-output ACObased MUD designed for a BPSK modulated system supporting $K$ users, there is a $(2 \times K)$-element so-called route table portrayed as

where each column represents the two legitimate values of a specific user's transmitted bit. Every trial vector $\hat{\mathbf{v}}_{i}^{\prime}$ comprising $K$ bits which are either +1 or -1 constitutes a possible route that might be pursued by the artificial ants. The conventional hard-output ACO-based MUD's aim is to find the ML vector without exhaustively searching over the $2^{K}$ possible combinations.

The searching processes of both ACO-based MUDs are extended from an initial stage decided by the intrinsic affinity derived from the MF output. This initial stage is then optimized by updating the pheromone, which is derived from the likelihood value of every route containing $K$ columns or nodes in the searching pool during each search iteration.

During the $n$th iterative search stage invoked by the ACOMUD, the node $v_{k}^{\prime}=+1$ or -1 is selected with a probability of [1]:

$$
p_{k, v_{k}^{\prime}}^{(n)}=\frac{\tau_{k, v_{k}^{\prime}}^{(n) \alpha} \eta_{k, v_{k}^{\prime}}^{\beta}}{\sum_{j \in\{+1,-1\}} \tau_{k, j}^{(n) \alpha} \eta_{k, j}^{\beta}},
$$


where $\tau_{k, v_{k}^{\prime}}^{(n)}$ represents the pheromone related to the node $v_{k}^{\prime}=$ $b \in\{+1,-1\}$ during the $n$th iteration, while $\eta_{k, v_{k}^{\prime}}$ represents the intrinsic affinity of the node. Furthermore, $\alpha$ and $\beta$ are parameters of the ACO algorithm as defined in [15]-[17].

The searching process ends at the $N_{e}$ th iteration, if all the vectors in the search pool of the current iteration become identical or if we reached $N_{e}=N$, where $N$ is the maximum number of affordable search iterations. The hard-output of the ACO-MUD is then the specific vector having the highest likelihood value in all the search pools created throughout the $N_{e}$ iterations. If we assume that there are $M$ number of ants, then the maximum number of likelihood value estimations is $N \cdot M$, which is only a small fraction of $2^{K}$, encountered in the ML detector, especially when $K$ is high.

For more details on the conventional ACO based MUD, the interested readers are referred to [15]-[17].

\section{Soft-Output Ant Colony Optimization Based MULTIUSER DETECTOR}

The hard decision criterion should be based on selecting the signal corresponding to the trial vector $\hat{\mathbf{v}}_{i}^{\prime}$ maximizing the $a$ posteriori probability $p\left(\hat{\mathbf{v}}_{i}^{\prime} \mid \mathbf{r}, \mathbf{H}\right)$ based on the observation of the received signal vector $\mathbf{r}$ at the BS and the CHTF matrix H. Using Bayes' rule, the a posteriori probability may be expressed as [27]:

$$
P\left(\hat{\mathbf{v}}_{i}^{\prime} \mid \mathbf{r}, \mathbf{H}\right)=\frac{p\left(\mathbf{r} \mid \hat{\mathbf{v}}_{i}^{\prime}, \mathbf{H}\right) P\left(\hat{\mathbf{v}}_{i}^{\prime}\right)}{p(\mathbf{r})}, \quad i=1,2, \ldots, 2^{K},
$$

where $p\left(\mathbf{r} \mid \hat{\mathbf{v}}_{i}^{\prime}, \mathbf{H}\right)$ is the conditional Probability Density Function (PDF) of the observed received signal vector $\mathbf{r}$, given that $\hat{\mathbf{v}}_{i}^{\prime}$ was transmitted via the DS-CDMA multiuser UL channel $\mathbf{H}$, and $P\left(\hat{\mathbf{v}}_{i}^{\prime}\right)$ is the a priori probability of the $i$ th $K$-user bitcombination considered. Therefore, the soft-bit value or the Log-Likelihood Ratio (LLR) associated with the $k$ th user can be formulated as [28]:

$$
L_{k}=\ln \frac{P\left(v_{k}^{\prime}=+1 \mid \mathbf{r}, \mathbf{H}\right)}{P\left(v_{k}^{\prime}=-1 \mid \mathbf{r}, \mathbf{H}\right)} .
$$

Note that the probability of $P\left(v_{k}^{\prime}=b \in\{+1,-1\} \mid \mathbf{r}, \mathbf{H}\right)$ is given by the sum of the probabilities $P\left(\hat{\mathbf{v}}^{\prime} \mid \mathbf{r}, \mathbf{H}\right)$ for all those vectors $\hat{\mathbf{v}}^{\prime} \in \mathcal{V}_{b}$, for which the $k$ th bit is $b$. Thus Eq. (13) can be further expressed as:

$$
L_{k}=\ln \frac{\sum_{\hat{\mathbf{v}}^{\prime} \in \mathcal{V}_{+1}} P\left(\hat{\mathbf{v}}^{\prime} \mid \mathbf{r}, \mathbf{H}\right)}{\sum_{\hat{\mathbf{v}}^{\prime} \in \mathcal{V}_{-1}} P\left(\hat{\mathbf{v}}^{\prime} \mid \mathbf{r}, \mathbf{H}\right)} .
$$

The denominator of Eq. (12) may be expressed as [27]:

$$
p(\mathbf{r})=\sum_{i=1}^{2^{K}} p\left(\mathbf{r} \mid \hat{\mathbf{v}}_{i}^{\prime}, \mathbf{H}\right) P\left(\hat{\mathbf{v}}_{i}^{\prime}\right),
$$

which is independent of the particular $K$-bit-combination transmitted or for the specific $K$-bit string $i=1, \ldots, 2^{K}$ being considered. Furthermore, if the a priori probabilities $P\left(\hat{\mathbf{v}}_{i}^{\prime}\right)$ are equal for all the $K$-bit strings $i=1,2, \ldots, 2^{K}$, Eq. (13) can be further expressed as:

$$
L_{k}=\ln \frac{\sum_{\hat{\mathbf{v}}^{\prime} \in \mathcal{V}_{+1}} p\left(\mathbf{r} \mid \hat{\mathbf{v}}^{\prime}, \mathbf{H}\right)}{\sum_{\hat{\mathbf{v}}^{\prime} \in \mathcal{V}_{-1}} p\left(\mathbf{r} \mid \hat{\mathbf{v}}^{\prime}, \mathbf{H}\right)} .
$$

Note that in Eq. (2), $\mathbf{r}$ is a random sample of the $N_{s}$-dimensional multi-variate complex Gaussian distribution, where the vector of mean value is $\mathbf{C H v}^{\prime}$, and the $\left(N_{s} \times N_{s}\right)$ dimensional covariance matrix $\mathbf{R}_{\mathbf{n}}$ is given by [29]:

$$
\mathbf{R}_{\mathbf{n}}=E\left\{\mathbf{n} \mathbf{n}^{H}\right\}=\sigma_{n}^{2} \mathbf{I},
$$

where $\mathbf{I}$ is the identity matrix. The noise encountered at the BS during the different the chip durations is assumed to be uncorrelated. Hence, the above-mentioned multi-variate complex Gaussian distribution can be described by [30]:

$$
p\left(\mathbf{r} \mid \hat{\mathbf{v}}^{\prime}, \mathbf{H}\right)=\frac{\exp \left\{-\left(\mathbf{r}-\mathbf{C H} \hat{\mathbf{v}}^{\prime}\right)^{H} \mathbf{R}_{\mathbf{n}}{ }^{-1}\left(\mathbf{r}-\mathbf{C H} \hat{\mathbf{v}}^{\prime}\right)\right\}}{\sqrt{2 \pi\left|\mathbf{R}_{\mathbf{n}}\right|^{N_{s}}}} .
$$

By substituting Eq. (17) into Eq. (18), we have [27]:

$$
p\left(\mathbf{r} \mid \hat{\mathbf{v}}^{\prime}, \mathbf{H}\right)=\frac{1}{{\sqrt{2 \pi \sigma_{n}^{2}}}^{N_{s}}} \exp \left\{-\frac{1}{2 \sigma_{n}^{2}}\left\|\mathbf{r}-\mathbf{C H} \hat{\mathbf{v}}^{\prime}\right\|^{2}\right\} .
$$

The previously mentioned likelihood value $D\left(\hat{\mathbf{v}}^{\prime}\right)$ associated with the vector $\hat{\mathbf{v}}^{\prime}$ is derived from the Euclidean distance $\| \mathbf{r}-$ $\mathbf{C H} \hat{\mathbf{v}}^{\prime} \|^{2}$ and is formulated as [27]:

$$
D\left(\hat{\mathbf{v}}^{\prime}\right)=2 \Re\left\{\hat{\mathbf{v}}^{\prime H} \mathbf{y}\right\}-\hat{\mathbf{v}}^{\prime H} \mathbf{R} \hat{\mathbf{v}}^{\prime} .
$$

Thus, Eq. (16) can be further expressed as:

$$
L_{k}=\ln \frac{\sum_{\hat{\mathbf{v}}^{\prime} \in \mathcal{V}_{+1}} \frac{1}{{\sqrt{2 \pi \sigma_{n}^{2}}}^{N_{s}}} \exp \left\{-\frac{1}{2 \sigma_{n}^{2}}\left\|\mathbf{r}-\mathbf{C H} \hat{\mathbf{v}}^{\prime}\right\|^{2}\right\}}{\sum_{\hat{\mathbf{v}}^{\prime} \in \mathcal{V}_{-1}} \frac{1}{{\sqrt{2 \pi \sigma_{n}^{2}}}^{N_{s}}} \exp \left\{-\frac{1}{2 \sigma_{n}^{2}}\left\|\mathbf{r}-\mathbf{C H} \hat{\mathbf{v}}^{\prime}\right\|^{2}\right\}} .
$$

To avoid the exponentially increasing computational complexity, the sums in the numerator and denominator of Eq. (21) are replaced by their most dominant term, i.e. by the highest term, as suggested in [29], yielding:

$$
L_{k} \approx-\frac{1}{2 \sigma_{n}^{2}}\left(\left\|\mathbf{r}-\mathbf{C H} \hat{\mathbf{v}}_{+1}^{\prime}\right\|^{2}-\left\|\mathbf{r}-\mathbf{C H} \hat{\mathbf{v}}_{-1}^{\prime}\right\|^{2}\right),
$$

where $\hat{\mathbf{v}}_{b \in\{+1,-1\}}^{\prime}$ represents the vector having the highest likelihood value in the set $\mathcal{V}_{b}$. For simplicity, the above equation can also be expressed as:

$$
L_{k} \approx-\frac{1}{2 \sigma_{n}^{2}}\left(\hat{v}_{k}^{\prime} \cdot \Omega_{k, \hat{v}_{k}^{\prime}}-\hat{v}_{k}^{\prime} \cdot \Omega_{k,-\hat{v}_{k}^{\prime}}\right),
$$

where $\hat{v}_{k}^{\prime}$ represents the estimated value of the $k$ th user's transmitted bit $v_{k}^{\prime}, \hat{\mathbf{v}}_{\hat{v}_{k}^{\prime}}^{\prime}$ denotes the vector having the highest likelihood value in the set $\mathcal{V}_{\hat{v}_{k}^{\prime}}$ and $\Omega_{k, \hat{v}_{k}^{\prime}}=\left\|\mathbf{r}-\mathbf{C H} \hat{\mathbf{v}}_{\hat{v}_{k}^{\prime}}^{\prime}\right\|^{2}$ represents the Euclidean distance between a legitimate received signal vector corresponding to a specific transmitted signal vector appropriately rotated by the channel and the actual received signal vector.

In order to calculate $\Omega_{k, \pm \hat{v}_{k}^{\prime}}$, all the $X\left(X \leqslant N_{e} \cdot M\right)$ number of mutually different vectors in the search pool are divided into two groups according to the value of the $k$ th bit of every vector. However, under certain circumstances all the $X$ number of vectors may have the same value $\hat{v}_{k}^{\prime}$ at the $k$ th 
bit position. In this case, an extra search iteration has to be carried out, where the $k$ th bit is fixed to $-\hat{v}_{k}^{\prime}$ throughout the search process.

Let us now characterize the achievable performance of the SISO ACO-based MUD.

\section{Simulation Result}

The DS-CDMA UL modem used in our simulations employed $N_{s}=31$-chip Gold codes to support $K=32$ users. The proposed soft-output ACO-based MUD was combined with a half-rate Convolutional Code (CC) having a constraintlength of $K_{c}=3$. The simulation parameters are summarized in Tab. I. For convenience, the common parameters shared by the soft and the conventional ACO-based MUDs have the same notations. For more details on the conventional hard-output ACO-based MUD, the interested reader is referred to [17].

TABLE I: Basic simulation parameters used in Section V.
\begin{tabular}{|c|l|l|}
\hline \multirow{4}{*}{$\begin{array}{c}\text { CC } \\
\text { parameters }\end{array}$} & Modem & BPSK \\
\cline { 2 - 3 } & Code rate & $1 / 2$ \\
\cline { 2 - 3 } & Constraint length & 3 \\
\cline { 2 - 3 } & Octal generator polynominal & $(031,027)$ \\
\hline \multirow{4}{*}{$\begin{array}{c}\text { SISO- } \\
\text { ACO } \\
\text { parameters }\end{array}$} & Initial pheromone & $\tau=0.01$ \\
\cline { 2 - 3 } & Evaporation rate & $\rho=0.5$ \\
\cline { 2 - 3 } & Number of ants & $M=10$ \\
\cline { 2 - 3 } & Number of iterations & $N=10$ \\
\cline { 2 - 3 } & Weight of pheromone & $\alpha=1$ \\
\cline { 2 - 3 } & Weight of intrinsic affinity & $\beta=6$ \\
\cline { 2 - 3 } & Weight for the elite ant & $\sigma=8$ \\
\hline Interleaver \\
parameters & Type & random \\
\cline { 2 - 3 } & size & $10^{4}$ bits \\
\hline
\end{tabular}

Fig. 2 shows that the DS-CDMA UL supporting $K=32$ users with the aid of $N_{s}=31$-chip Gold codes is capable of approaching the corresponding single-user system's BER, regardless, whether the soft-output or hard-output ACO-based MUD is used. However, the soft-output ACO-assisted DSCDMA scheme shows a significant SNR improvement compared to its hard-output ACO-assisted counterpart.

Fig. 3 shows that the complexity of the ACO-based MUD is only a fraction of that of the ML or Bayesian detector, again, regardless, whether hard- or soft-output aided detection is used. More quantatively, when the number of users reaches $K=32$, the complexity of either the hard-output or the softoutput ACO is a factor of $10^{8}$ lower than that of the optimum Bayesian detector.

\section{CONCLUSION}

In conclusion, the proposed soft-output ACO-based MUD is capable of approaching the single-user performance, when combined with a 1/2-rate convolutional code, as seen for the DS-CDMA UL supporting $K=32$ users by employing 31chip Gold sequences. The complexity of the soft ACO-based MUD based on Tab. I is a factor of $10^{8}$ lower than that of the Bayesian MUD.

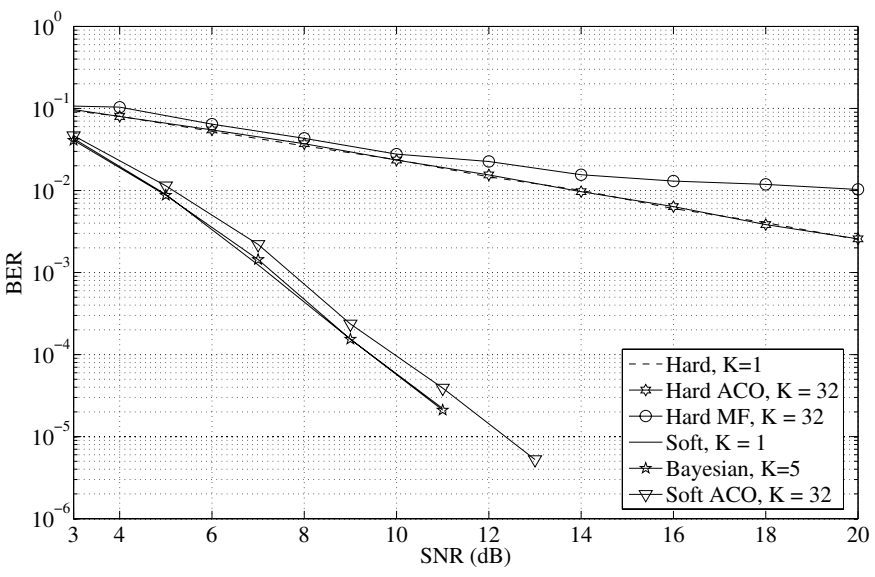

Fig. 2: BER versus SNR performance of the uplink DS-CDMA system for transmission over uncorrelated flat Rayleigh fading channels using $N_{c}=31$-chip Gold codes.

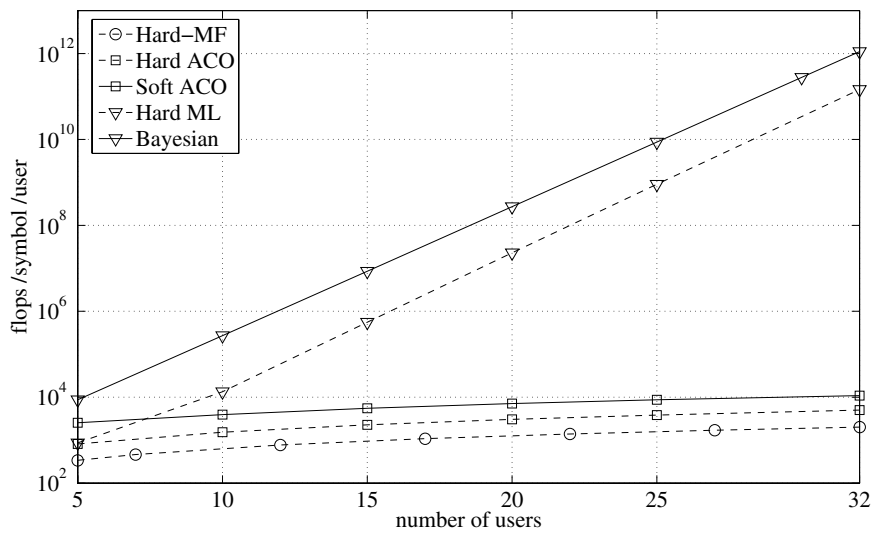

Fig. 3: Complexity per transmitted signal per user versus the number of users of the DS-CDMA system employing $N_{c}=31$-chip Gold codes.

\section{REFERENCES}

[1] A. Colorni, M. Dorigo and V. Maniezzo, "Distributed optimization by ant colonies," in Proceedings of the European Conference on Artificial Life, 1991, pp. 134-142.

[2] M. Dorigo, V. Maniezzo and A. Colorni, Ant system: an autocatalytic optimizing process. Technical report, Politechnico di Milano, Italy, no. $91-016,1991$. url = "citeseer.ist.psu.edu/119878.html"

[3] T. Stützle and H.-H. Hoos, "MAX-MIN Ant system," Future Generation Computer Systems, vol. 16, pp. 889-914, Jun. 2000.

[4] M. Dorigo, V. Maniezzo and A. Colorni, "Ant system: optimization by a colony of cooperating agents," IEEE Transactions on Systems, Man and Cybernetics, Part-B, vol. 26, pp. 29-41, Feb. 1996.

[5] M. Dorigo and L.-M. Gambardella, "Ant colony system: a cooperative learning approach to the traveling salesman problem," IEEE Transactions on Evolutionary Computation, vol. 1, pp. 53-66, Apr. 1997.

[6] E.-G. Talbi, O. Roux, C. Fonlupt and D. Robillard, "Parallel ant colonies for the quadratic assignment problem," Future Generation Computer Systems, vol. 17, pp. 441-449, Jan. 2001.

[7] A. Colorn, M. Dorigo, V. Maniezzo, M. Trubian, "Ant system for jobshop scheduling," JORBEL-Belgian Journal of Operations Research, Statistics and Computer Science, vol. 34, pp. 39-53, 1994.

[8] G.-D. Caro and M. Dorigo, "Mobile agents for adaptive routing," in Proceedings of IEEE Hawaii International Conference on System Sciences, vol. 7, 1998, pp. 74-83.

[9] K.-M. Sim and W.-H. Sun, "Ant colony optimization for routing and load-balancing: survey and new directions," IEEE Transactions on Systems, Man and Cybernetics, Part A, vol. 33, pp. 560-572, Sep. 2003. 
[10] P. Wang, X.-M. Gu and G.-L. Liu "Multi-QoS routing for LEO satellite networks," in Proceedings of IEEE International Conference on Advanced Communication Technology, vol. 1, 2007, pp. 728-731.

[11] S. L. Hijazi and B. Natarajan, "Near-optimal multiuser detection in asynchronous MC-CDMA via the ant colony approach," in Proceedings of the IEEE International Symposium on Wireless Pervasive Computing, vol. 1, 2007, pp. 274-279.

[12] S. L. Hijazi, A.J. Best B. Natarajan and S. Das, "Ant-colony based optimal MC-CDMA multiuser detector," in Proceedings of the IEEE International Conference on Wireless and Mobile Computing, Networking and Communications, vol. 1, 2005, pp. 128-132.

[13] S. L. Hijazi, B. Natarajan and S. Das, "An ant-colony algorithm for multi-user detection in wireless communication systems," in Proceedings of Genetic and Evolutionary Computation Conference, 2005, pp. 21212126.

[14] S. L. Hijazi and B. Natarajan, "Novel low-complexity DS-CDMA multiuser detector based on ant colony optimization," in Proceedings of IEEE Vehicular Technology Conference, vol. 3, 2004, pp. 1939-1943.

[15] J.-J. Lai and J.-K. Lain, "Antenna-diversity-assisted ant-colony-based multiuser detection for DS-CDMA systems," in Proceedings of the IEEE International Workshop on Cellular Neural Networks and Their Applications, vol. 3, 2005, pp. 106-109.

[16] C. Xu, L.-L. Yang and L. Hanzo, "Ant-colony-based multiuser detection for MC DS-CDMA systems," n Proceedings of the IEEE Vehicular Technology conference, Fall, pp. 960-964, 2007.

[17] C. Xu, B. Hu, L.-L. Yang and L. Hanzo, "Ant-colony-based multiuser detection for multi-functional antenna array assisted MC DS-CDMA systems," IEEE Transactions on Vehicular Technology, vol. 57, pp. 658663, Jan. 2008.

[18] M. Dorigo, G.-D. Caro and L.-M. Gambardella, "Ant algorithms for distributed discrete optimition," in Proceedings of the International Conference on Artificial Life, vol. 5, 1999, pp. 137-172.

[19] T. Stützle and H.-H. Hoos, "The MAX-MIN ant system and local search for the traveling salesman problem," in Proceedings of IEEE International Conference on Evolutionary Computation, 1997, pp. 309314.

[20] M. Birattari, P. Pellegrini and M. Dorigo, "On the invariance of any colony optimization," to appear in IEEE Transactions on Evolutionary Computation.

[21] T. C. Fogarty, "Using the genetic algorithm to adapt intelligent systems," in Proceedings of the IEE Colloquium on Symbols Versus Neurons, vol. 12, 1990, pp. 4/1-4/4.

[22] M. Jiang, S.-X. Ng, L. Hanzo, "Hybrid iterative multiuser detection for channel coded space division multiple access OFDM systems," IEEE Transactions on Vehicular Technology, vol. 55, pp. 115-127, Jan. 2006.

[23] C. Berrou, A. Glavieux, and P. Thitimajshima, "Near shannon limit error-correcting coding and decoding: turbo codes," in Proceedings of the IEEE International Conference on Communications, vol. 2, 1993, pp. 1064-1070.

[24] C. Berrou and A. Glavieux, "Near optimum error correcting coding and decoding: turbo-codes," IEEE Transactions on Communications, vol. 44, pp. 1261-1271, Oct. 1996.

[25] L. Hanzo, T.-H. Liew and B.-L. Yeap, Turbo Coding, Turbo Equalisation and Space-Time Coding for Transmission over Fading Channels. Chichester, U.K.: Wiley, 2002.

[26] B. Sklar, "A primer on turbo code concepts," IEEE Communications Magazine, vol. 35, pp. 94-102, Dec. 1997.

[27] J.G. Proakis, Digital Communications Fourth Edition. McGraw-Hill, 2000.

[28] T. K. Moon and W. C. Stirling, Mathematical Methods and Algorithms for Signal Processing. New Jersey, U.S.A.: Prentice Hall, 2002.

[29] L. Hanzo, M. Munster, B. J. Choi and T. Keller, OFDM and MC-CDMA for Broadband Multi-user Communications, WLANs and Broadcasting. Chichester, U.K.: Wiley, 2003.

[30] S. Kay, Fundamentals of Statistical Signal Processing, Estimation Theory. New Jersey, U.S.A.: Prentice Hall, 1993. 